\title{
MRI features in the non-traumatic spinal cord injury patients presenting at the Korle Bu Teaching Hospital, Accra
}

\section{Hafisatu Gbadamosi, Yaw B. Mensah and Samuel Asiamah}

Ghana Med J 2018; 52(3): 127-132 doi: http://dx.doi.org/10.4314/gmj.v52i3.4

\section{Department of Radiology, Korle Bu Teaching Hospital, Accra, Ghana}

\author{
Corresponding author: Dr Hafisatu Gbadamosi \\ E-mail: hafigbadamosi@yahoo.com \\ Conflict of interest: None declared
}

\section{SUMMARY}

Background: Neurological limb deficit due to non-traumatic myelopathy is a disabling and distressing neurological condition. In recent time Magnetic Resonance Imaging (MRI) has proven to be the ultimate imaging modality for evaluating pathologies of the spinal cord.

Objective: To describe the Magnetic Resonance Imaging (MRI) features of patients with Non-Traumatic Spinal Cord Injury evaluated at the Korle Bu Teaching Hospital.

Methods: A descriptive cross-sectional study was carried out at the Korle Bu Teaching Hospital (KBTH), Accra, Ghana.

Results: Out of a total of 141 MRI's evaluated $60.3 \%$ were males and $39.7 \%$ female. The majority of the respondents $85.1 \%$ had paraparesis/paraplegia,13.5\% had quadriparesis/quadriplegia, $1.4 \%$ had weakness in one upper limb and both lower limbs. The commonest MRI features of NTSCI recorded was due to degenerative disease of the spine $75.9 \%$, spinal metastases $5.7 \%$, Pott's/pyogenic spondylitis $3.5 \%$, demyelinating disease $2.8 \%$ and primary spinal tumours $2.8 \%$.

Conclusion: The commonest MRI findings in the study population were due to degenerative disease of the spine, followed by spinal metastases and infective spondylitis.

Funding: Not declared

Keywords: paraparesis, paraplegia, tetraparesis, tetraplegia, Magnetic Resonance Imaging

\section{INTRODUCTION}

Spinal cord injury is a debilitating medical condition. A recent review reported the incidence of Traumatic Spinal Cord Injury (TSCI) worldwide to be between 10.4 and 83 cases per million per year. ${ }^{1}$ In comparison to TSCI, there is relatively little research into Non-Traumatic Spinal Cord Injury (NTSCI) in spite of the fact that several known conditions can lead to NTSCI. Common aetiologies of NTSCI include infection, ischemia, primary or metastatic tumours and degenerative disease of the spine. $^{2,3}$

Non-Traumatic Spinal Cord Injury may present as paraparesis /paraplegia or tetraparesis/ tetraplegia. Paraparesis is defined as partial loss and paraplegia as complete loss of function of both lower limbs; tetraparesis is the partial loss of and tetraplegia complete loss of function of both upper limbs and lower limbs as a result of disease or injury to the spinal cord, spinal roots, and peripheral nerves or due to myopathies. It could also result from certain parasagittal intracranial lesions. ${ }^{4}$
Clinically, the diagnosis of myelopathy depends on the ability to localize the clinical findings to the spinal cord, instead of to the brain or peripheral nervous system and in some cases to a particular segment of the spinal cord. As such the antecedent clinical syndrome and other details of the patient's course are helpful and would ultimately contribute significantly to localizing the pathology on imaging.

In the same vein, the clinical information provided by the referring doctors is vital for radiological interpretation of NTSCI especially in acute myelopathy where early treatment may reverse symptoms. This helps to elucidate the likely pathological aetiology from a tall list of what can have very similar imaging features. Of extreme importance are the onsets of symptoms, patients' age and sex which may also help to narrow the differential diagnosis. Accurate clinical examination will again help determine the likely anatomical location of lesions within the cord and provide clues to the nature of the underlying pathology. 
Knowledge of this enables better-targeted imaging and interpretation of incidental findings in the wrong location. ${ }^{5,6}$ In the not too distant past, studies on myelopathy were investigated solely by conventional radiography and myelography, ${ }^{7,8,9}$ Over the past two decades, however, MRI has proven to be the premier imaging modality for the evaluation of myelopathy. Consequently, older modalities such as myelography are now limited to the evaluation of cord contour. ${ }^{10}$ While myelography demonstrates nerve root take off and thecal sac compression, it is however neither specific nor sensitive.

Computed tomography (CT) on the other hand continues to be useful for the evaluation of the bony spine for example, in cases of trauma, spondylosis, or tumour, but it is less sensitive for demonstrating intrinsic lesions of the cord. Undoubtedly MRI has revolutionized the imaging of myelopathy; not only is MRI more sensitive than CT or myelography for the detection of spinal cord disease, it is also more specific for the characterization of intrinsic cord disease. ${ }^{11}$ MRI has several advantages over CT and it dominates in the elegance with which it delineates diseases of the spine and spinal cord. MRI facilitates 3-dimensional imaging; it has excellent imaging of the neural elements, shows increased accuracy in diagnosing intrinsic cord disease, as well as being a noninvasive, radiation-free procedure; It is also without major side effects. ${ }^{12}$

The impact of spinal myelopathy on the socioeconomic status of patients and their families is quite severe. Giving the complexity of the disease process a multidisciplinary approach is needed to manage the condition especially in the early stages of the disease. The early use of MRI would help to arrive at a definite diagnosis, thereby contributing to early treatment and to the arrest of disease progression in some cases. This study thus sought to describe the MRI features of patients with Non-Traumatic Spinal Cord Injury evaluated at the Korle Bu Teaching Hospital as well as the attendant patient demographics.

\section{METHODS}

This was a descriptive cross-sectional prospective study carried out at the Korle Bu Teaching Hospital (KBTH), Accra, Ghana from June to November 2014. The study was conducted in the Radiology Department, Neurosurgical Unit of the Department of Surgery and the Neurology Unit of the Department of Medicine and Therapeutics of the Korle Bu Teaching Hospital (KBTH).

KBTH is the largest hospital in Ghana and boasts of wellestablished neurology and neurosurgical departments. It is located in Accra, the capital city of Ghana, which is largely metropolitan. As such, most of the referrals from the southern sector of the country and parts of the West African sub-region are seen in this hospital.
It also serves as a centre for training residents of the West Africa and the Ghana Postgraduate Medical and Surgical Colleges.

Patients sent to the Radiology Department of KBTH for MRI studies of the spine who met the study criteria and those with similar presentations attending the neurology and neurosurgery clinics with previously acquired MRI were included in the study. The sample size was calculated using the Leslie Kish/ Fisher formula for cross-sectional studies. ${ }^{13}$ Results of a study in Yaoundé, Cameroon put the incidence of Non-traumatic myelopathies at 9.7. ${ }^{14}$ Using this information from the sub-region, a sample size of 135 participants was estimated. Using a non-respondent rate of $6 \%$ a total of 141 patients were interviewed.

Written informed consent was sought from all the patients. With the help of a semi-structured questionnaire the relevant patient demographic information was obtained.

Images obtained from MRI studies from the participants were evaluated and documented.

Exclusion criteria:

1. Patients presenting with Traumatic Limb Neurological Deficit

2. Non-Ghanaians

3. Individuals aged less than 13 years

\section{Imaging Protocol}

The standard approach to imaging a suspected intradural intramedullary mass used for the study consisted of;

1. T1 weighted Fast Spine Echo (FSE) sagittal and axial, T2 weighted FSE sagittal and axial, T1 weighted FSE with contrast; sagittal and axial, with both planes fat saturated (especially when there was foraminal extension or when there was an extradural process).

2. Sagittal images were $5.0 \mathrm{~mm}$ thick with a $0.5 \mathrm{~mm}$ slice gap. The field of view (FOV) of the area of interest used was $24 \mathrm{~cm}$ in cervical spine and $32 \mathrm{~cm}$ in lumbosacral spine. In the thoraco-lumbar spine, a large FOV $(34 / 36 \mathrm{~cm})$ was used.

3. Axial images were $5.0 \mathrm{~mm}$ thick with a $0.5 \mathrm{~mm}$ slice gap and obtained using FSE sequences. Technical parameters included $16^{\circ}$ flip angle, minimum TR/TE, $224 \AA \sim 320$ matrix and two excitations in T1WI and one excitation in T2WIs. The TE used was less than $15 \mathrm{~ms}$ in T1WI and up to $100 \mathrm{~ms}$ in T2WIs.

For suspected degenerative disc disease, the following protocol was used;

1. Coronal, Cervical, Thoracic and lumbar region; Sagittal region; Sagittal T1 weighted (3:09 - 4:50 minutes)

2. Sagittal T2 weighted (1: $50-4.16$ minutes)

3. Axial FSE T2 weighted (4.26 - 7.60 minutes) 
In addition to a typical study for degenerative disc pathology additional sequences were done for the various aetiologies. For suspected Spinal tumours, demyelinating disease, syringomyelia and spinal infections;

1. Sagittal STIR (2.53 - 4.05 minutes)

2. Axial and Sagittal T1 weighted fat suppression with Gadolinium enhancement (Axial 2.59 - 6.36 minutes and Sagittal $2.44-5.06$ minutes) in a dose of 0.1 $\mathrm{mmol} / \mathrm{kg}$

3. Diffusion weighted imaging sequence was used for suspected spinal cord infarcts (3.4 minutes)

4. Coronal T2 weighted sequences for scoliosis $2.02-$ 4.42 minutes.

\section{Ethical Consideration}

Ethical clearance was sought from and granted by the Research Ethics and Protocol Review Committee of the University of Ghana Medical School, MS-Et/M.10 P4.7/2013/2014

\section{RESULTS}

Out of the total 141 patients who took part in the study 85 (60.3\%) were male and 56 (39.7\%) were female. The age range of the study population was between 16 to 79 years. The mean age for the general study population was 56.4 years (SD 15.4), while that for males was 53.6 years (SD 16.4) and 60.3 years (SD 13.7) for females. The occupation of the study group was varied. They were grouped into farmer/fisherman, trader, artisan, professional, retired, student and 'others' (including nontraditional jobs such as domestic servants, truck pushers and cleaners) for easy interpretation.

Two groups had the highest numbers of respondents; these were the professional and trader groups comprising 40 patients (28.4\%), followed by nontraditional jobs, 34 patients (24.1\%), retired, 13 patients (9.2\%), artisan, 6 patients (4.3\%), then by the fisherman/farmer and student groups, each with 4 patients (2.8\%). A wide range of diagnoses were made on MRI as the underlying cause of the patients' weakness/paralysis.

The commonest diagnosis was degenerative disease of the spine with 107 patients (75.9\%) manifesting imaging features of this condition. This was followed distantly by spinal metastases in 8 patients (5.7\%), Pott's disease/pyogenic spondylitis in 5 patients (3.5\%), demyelinating disease in 4 patients $(2.8 \%)$ and syrinx in 4 patients $(2.8 \%)$ as shown in Table 1.
Table 1: MRI findings in patients

\begin{tabular}{|l|r|}
\hline Diagnosis & $\mathbf{n}(\%)$ \\
\hline Degenerative disease of the spine & $107(75.9)$ \\
\hline Pott's disease/Pyogenic spondylitis & $8(5.7)$ \\
\hline Demyelinating Disease & $5(3.5)$ \\
\hline Syrinx & $4(2.8)$ \\
\hline Primary malignant Extradural tumour & $4(2.8)$ \\
\hline Inflammatory/Autoimmune Condition & $2(1.4)$ \\
\hline Primary malignant bone tumour & $2(1.4)$ \\
\hline Other (Scoliosis, Kyphosis) & $2(1.4)$ \\
\hline Normal findings & $2(1.4)$ \\
\hline TOTAL & $5(3.5)$ \\
\hline
\end{tabular}

Concerning the prevalence of disease in the various age groups, most of the significant MRI findings were seen in patients who were over 60 years. They represented close to half of the cases of degenerative disease (43.9\%) and infective spondylitis (40\%); a quarter of cases of metastases and all the cases of inflammatory/ autoimmune disease. There were no patients with degenerative disease in the second and third decades. The only two cases of congenital aetiology i.e. scoliosis and kyphoscoliosis were in the second decade (Table 2).

The various Magnetic Resonance Images reviewed revealed that majority of findings, $54.7 \%$ were noted in the lumbar region while $21.3 \%$ were seen in the cervical region, $16.0 \%$ in the thoracic spine and $2.7 \%$ of the findings involved the entire spine. In $5.3 \%$ of the patients no abnormal MRI findings were seen.

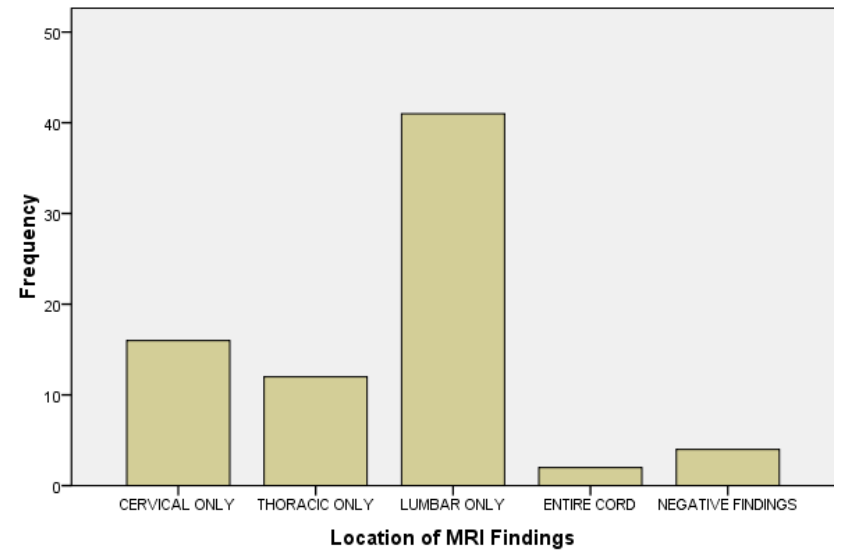

Figure 1: Location of various findings in the spine 
Table 2: Age distribution versus patients' diagnosis

\begin{tabular}{|l|c|c|c|c|c|c|c|}
\hline \multirow{2}{*}{ Diagnosis } & \multicolumn{7}{|c|}{ Age-Group (years) } \\
\cline { 2 - 8 } & $\mathbf{1 3 - 2 0}$ & $\mathbf{2 1 - 3 0}$ & $\mathbf{3 1 - 4 0}$ & $\mathbf{4 1 - 5 0}$ & $\mathbf{5 1 - 6 0}$ & $>\mathbf{6 1}$ & TOTAL \\
\hline Degenerative disease of the spine & 0 & 0 & 2 & 19 & 39 & 47 & $\mathbf{1 0 7}$ \\
\hline Metastases & 0 & 2 & 0 & 4 & 0 & 2 & $\mathbf{8}$ \\
\hline Pott's disease/Pyogenic spondylitis & 0 & 0 & 0 & 1 & 2 & 2 & $\mathbf{5}$ \\
\hline Demyelinating Disease & 0 & 2 & 0 & 2 & 0 & 0 & $\mathbf{4}$ \\
\hline Syrinx & 0 & 0 & 2 & 2 & 0 & 0 & $\mathbf{4}$ \\
\hline Inflammatory/Autoimmune Condition & 0 & 0 & 0 & 0 & 0 & 2 & $\mathbf{2}$ \\
\hline Primary malignant Extradural tumour & 0 & 0 & 0 & 2 & 0 & 0 & $\mathbf{2}$ \\
\hline Primary malignant bone tumour & 0 & 0 & 0 & 2 & 0 & 0 & $\mathbf{2}$ \\
\hline Others & 2 & 0 & 0 & 0 & 0 & 0 & $\mathbf{2}$ \\
\hline Normal Finding & 0 & 0 & 1 & 2 & 2 & 0 & $\mathbf{5}$ \\
\hline TOTAL & $\mathbf{2}$ & $\mathbf{4}$ & $\mathbf{5}$ & $\mathbf{3 4}$ & $\mathbf{4 3}$ & $\mathbf{5 3}$ & $\mathbf{1 4 1}$ \\
\hline
\end{tabular}

\section{DISCUSSION}

Our study recorded more male patients, 60.35\% compared to $39.7 \%$ females. However, almost all the common conditions diagnosed on MRI did not show any significant gender predominance. This trend could be explained by the fact that although there may be many affected women, the patriarchal practices in many developing countries such as Ghana, make some women still rely on male approval before seeking medical care leading to delayed hospital attendance or none at all. This is corroborated by a study on the spectrum of non-traumatic myelopathies in Ethiopia, a country with similar societal norms which recorded a male to female ratio of 1.7 , only slightly higher than the 1.5 male to female ratio obtained in this study. ${ }^{15}$

The mean age for this study's population was 56.4 years (SD 15.4). This was higher than a similar study conducted in South-eastern Nigerian on the Profile and outcome of non-traumatic paraplegia in $\mathrm{Kano}^{16}$, while it had a similar age range (16 and 76 years), it had a lower mean age of 40 years (SD 15.3) years. This relatively younger mean age of the study population can be explained by the findings of conditions such as Pott's disease (44.9\%) and transverse myelitis (11.1\%) which are relatively more common in the younger age group; these two conditions were incidentally the two most common aetiologies of their study population. On the other hand, our study demonstrated a preponderance of conditions typically seen more commonly in the older age group such as degenerative disease of the spine (76\%) and metastases (6\%).

Another reason that can be adduced for the differences in the KBTH and Kano studies is that while the Nigerian study used x-rays for all their patients and MRI for only
$26 \%$ of their study population, our study used only MRI much like the situation in the Rawalpindi, Pakistan study $^{17}$, which also demonstrated their commonest aetiologies as shown on MRI as being attributed to degenerative myelopathy, infective and neoplastic pathology.

Another study in Zimbabwe, Parry $\mathrm{O}$ et al supported our findings by showing that their top two aetiologies of NTSCI included neoplasm as their commonest (28\%) followed by tuberculosis (27\%). ${ }^{18}$ Both studies also demonstrated that neoplasms were commoner in the lower thoracic and upper lumbar region. In spite of the fact that a reasonable number of suspected Pott's disease is seen in the clinical setting, the number of patients manifesting these findings in our study unlike the Nigerian study was low.

One reason for this finding is the fact that most of these patients may have manifested significant features of Pott's disease on plain radiography, which did not warrant a need for further evaluation with MRI in which case clinicians probably went ahead to treat without the need for MRI. Secondly most of these patients come from low socioeconomic backgrounds and may have been unable to afford the high cost of MRI imaging even if it was clinically indicated.

A study in Yaoundé by Lekoubou Looti et $\mathrm{al}^{14}$ showed a relatively higher proportion of Pott's disease compared to our study, but with numbers similar to the Kano, Nigerian study. ${ }^{16}$ It also showed a higher proportion of spinal metastases of $12 \%$ compared to $8 \%$ in this study and $4 \%$ in the Kano study. The Yaoundé study was a retrospective one conducted over a 6-year period and thus being significantly different in methodology. 
The researchers also had access to patients' laboratory workup including CSF analysis and even relied on a diagnosis of TB being made after a positive response to anti tuberculous treatment in some cases. Just like the Kano study, they used plain radiography as one of the imaging modalities while conventional and CT myelography replaced MRI. This may have accounted for the Yaoundé study having a relatively higher proportion of Pott's disease unlike our study.

Again No MRI studies were employed in the Yaoundé study. While their percentage of tuberculosis spondylitis cases was relatively higher than in our study the possibility of underdiagnoses of the other causes of myelopathy e.g. spondylotic myelopathy and demyelinating disease may have inadvertently occurred in the Yaoundé study. Moore et al, ${ }^{19}$ conducted a prospective study in Liverpool to evaluate the causes of non-traumatic spastic paraparesis and tetraparesis in 585 patients over a three-year period using a battery of clinical, myelographic and MRI studies of the spine.

Their age profile demonstrated a peaking of multiple sclerosis in the 40th decade with the proportions of cervical spondylotic myelopathy, neoplasm and motor neuron disease continuing to rise until the 60 - 69-year age group. Our study despite the fact that it spanned a relatively shorter period showed similar findings with the study by Moore et al, with approximately $44 \%$ of all the cases of degenerative disease of the spine being in the older than 60 age brackets and approximately half the cases of spinal metastases being within the 41-50 age range. Again, our study also showed that $50 \%$ of the cases with features of demyelinating disease were also in the 41-50-year age group.

For about $27.4 \%$ of the patients in the Liverpool study the diagnoses were uncertain. This study also revealed that $3.5 \%$ of our study population had normal MRI findings . These scenarios often arise due a few reasons including; insufficient clinical history, difficulty in following up with patients' primary physician, as well the absence of documentation of the patients' laboratory work up which may have been required to arrive at a definite diagnosis as well as the remote possibility that the wrong spinal segment was imaged in the absence of adequate clinical information. Recognizing the crucial role clinical history and dedicated patient examination play in image interpretation is vital.

The situations of inadequate information provided to the radiologist are not uncommonly encountered. The occurrence of uncertainty in diagnosis should serve as an important caution to clinicians to recognize the importance of clinician-radiologist collaboration, with total disclosure of clinical information to the radiologist to ensure appropriate diagnosis.

\section{CONCLUSION}

MRI was helpful in elucidating the various aetiology of non-traumatic limb weakness. The commonest MRI diagnosed cause of non-traumatic spinal cord injury in our study was degenerative disease of the spine. This was followed by spinal metastases and infective spondylitis. These findings are similar to those of other parts of Africa.

\section{REFERENCES}

1. Wyndaele M, Wyndaele JJ. Incidence, prevalence and epidemiology of spinal cord injury: what learns a worldwide literature survey? Spinal Cord 2006;44(9):523-9

2. McKinley O, Seel T, Hardman T. Non-traumatic spinal cord injury: incidence, epidemiology and functional outcome. Archives of Physical Medicine \& Rehabilitation. 1999;80 (6): 619-23

3. Sadasivan KK, Reddy RP, Albright JA. The natural history of cervical spondylotic myelopathy. Yale $J$ Biol Med 1993; 66:235-42

4. Venkatraman S, Dhamija RM, Sanchetee PC, et al. Progress in Clinical Neurosciences Neurology India 1990; 6:275-97.

5. Bradley, W.G. Myelopathy Applied Radiology 2001; 30(8):

6. Sheerin F, Collison K, Quagbebeur G. Magnetic resonance imaging of acute intramedullary myelopathy: radiological differential diagnosis for the on-call radiologist Clinical Radiology 2009;64 (1): 8 -9

7. Jain AP, Gupta OP, Jajoo UN. A study of some prognostic factors in acute transverse myelitis. $J$ Assoc Physicians India1983; 31:497-99.

8. Singhal B S.Non-compressive myelopathies with special reference to demyelinating diseases in Indian context. J Assoc Physicians India 1984; 32:509-12.

9. Venkatraman S, Dhamija RM, Sanchetee PC, et al. Progress in Clinical Neurosciences Neurology India 1990; 6:275-97.

10. Jacob A, Weinshenker BG.An approach to the diagnosis of acute transverse myelitis. Semin Neurol. 2008 Feb;28 (1):105-20.

11. New PW, Rawicki HB, Bailey MJ.Non-traumatic spinal cord injury: demographic characteristics and complications. Arch Phys Med Rehabil 2002; 83: 996- 1001

12. Bell GR, Ross JS. Diagnosis of nerve root compression: Myelography, Computed Tomography and MRI. Orthopedic Clinic North America 1992;23:405-419 
13. Kish Leslie et al. Survey Sampling. John Wiley and Sons NY. 1965.

14. Looti A Z, Kengne A P, Djentcheu V et al. Patterns of non-traumatic myelopathies in Yaounde'(Cameroon): a hospital-based study Neurol Neurosurg Psychiatry 2010;81:768-770

15. N J Fidèle N.J. Amanuel A. Spectrum of non-traumatic myelopathies in Ethiopian patients: hospitalbased retrospective study Spinal Cord 2016;54:604608

16. Owolabi L.F., Ibrahim A et al. Profile and outcome of non-traumatic paraplegia in Kano, northwestern Nigeria. An Afr Med 2011;10(2):86-90
17. Ahmed N, Akram $\mathrm{H}$ et al. Role of MRI in differentiating various causes of non-traumatic paraparesis and tetraparesis. J Coll Physicians Surg Pak 2004 Oct;14(10): 596-600.

18. Parry O. Bhebhe E. Levy L.F. Non-traumatic paraplegia [correction of paraplegis] in a Zimbabwean population--a retrospective survey. Cent Afr J Med. 1999;45(5):114-9.

19. Moore P. Blumhardt D. A prospective Survey of the causes of non-traumatic spastic paraparesis and tetraparesis in 585 patients Spinal Cord 1997;35: 361-367 